\title{
Review
}

\section{Review of quantitative measurements of upper limb movements in hemiplegic cerebral palsy}

\author{
Ellen Jaspers ${ }^{\mathrm{a}, *}$, Kaat Desloovere ${ }^{\mathrm{a}, \mathrm{b}}$, Herman Bruyninckx ${ }^{\mathrm{c}}$, Guy Molenaers ${ }^{\mathrm{d}}$, \\ Katrijn Klingels ${ }^{a}$, Hilde Feys ${ }^{a}$ \\ a Department of Rehabilitation Sciences, Faculty of Kinesiology and Rehabilitation Sciences, Katholieke Universiteit Leuven, Belgium \\ ${ }^{\mathrm{b}}$ Clinical Motion Analysis Laboratory, CERM, University Hospital of Pellenberg, Belgium \\ ${ }^{c}$ Department of Mechanical Engineering, Faculty of Engineering, Katholieke Universiteit Leuven, Belgium \\ ${ }^{\mathrm{d}}$ Department of Musculoskeletal Sciences, Faculty of Kinesiology and Rehabilitation Sciences, Katholieke Universiteit Leuven, Belgium
}

A R T I C L E I N F O

Article history:

Received 2 September 2008

Received in revised form 24 June 2009

Accepted 7 July 2009

\section{Keywords:}

Hemiplegia

Cerebral palsy

Upper limb

Movement analysis

\begin{abstract}
A B S T R A C T
This review provides an overview of results found in literature on objective measurements of upper limb movements in children with hemiplegic cerebral palsy (HCP). Seventeen articles were selected following a systematic search. Analysed tasks varied from simple reaching and gross motor functions to complex, fine motor tasks. Spatiotemporal characteristics have been extensively studied and longer movement durations, slower movement speed and reduced trajectory straightness at the affected upper limb, compared to the non-affected side or healthy children, were most frequently reported. Joint kinematics has been far less studied. The limited data confirm the clinical impression of children with HCP using less elbow extension and supination to reach for an object, which is compensated by increased trunk flexion. Increased trunk involvement was also reported during gross motor functions. Although threedimensional (3D) movement analysis seems promising to provide additional insights in the pathological upper limb movements observed in HCP, future standardisation of the entire protocol is crucial. No consensus exists on the procedures for data collection, processing, analysing and reporting of results, or what upper limb tasks should be assessed. The International Society of Biomechanics recently proposed recommendations on the definition of upper limb joint coordinate systems and rotation sequences. These guidelines were not yet applied in these studies. Although the diverse methodological approaches used in the studies complicate the comparison of published results, some general conclusions could be drawn. A further standardisation of the protocol for 3D upper limb movement analysis will provide the foundation for comparable and repeatable results and eventually facilitate the selection and planning of treatment interventions.
\end{abstract}

(c) 2009 Elsevier B.V. All rights reserved.

\section{Introduction}

Hemiplegia is the most common form of cerebral palsy (CP) among children born at term and second to diplegia among preterm infants [1]. Children with hemiplegic CP (HCP) are faced with various motor and sensory impairments, e.g. muscle weakness, spasticity, lack of selective motor control and sensory disturbances, with the upper limb more involved in about half of the children [2]. These upper limb impairments contribute to the difficulties experienced when reaching, grasping and manipulating objects [2-4]. Deficiencies in one or more of these basic functions hinder proper performance of activities of daily living (ADL) and as

\footnotetext{
* Corresponding author at: Tervuursevest 101, 3001 Leuven, Belgium. Tel.: +32 16 329117; fax: +32 16329197 .

E-mail address: ellen.jaspers@faber.kuleuven.be (E. Jaspers).
}

such may impact on independency and quality of life of the hemiplegic child.

Adequate treatment planning is imperative, though requires an extensive knowledge of all upper limb dysfunctions. A clinical assessment, combined with objective and quantitative measurements of the upper limb could provide the necessary insights. Current methods of clinical assessment evaluate both motor (range of motion, spasticity, muscle strength) and sensory impairments (exteroception, proprioception, two-point discrimination, stereognosis). Available clinical scales primarily assess the quality of upper limb movement during several functional tasks (e.g. Melbourne Assessment of Unilateral Upper Limb Function [5], Quality of Upper Extremity Skills Test [6], Shriners Hospital for Children Upper Extremity Evaluation-SHUEE [7]). These scales are widely used to evaluate upper limb function in HCP as they are easy to administer with a straightforward scoring system. However, the main disadvantage of these qualitative outcome 
measures is that they provide a subjective description of the upper limb task performance based on the assessor who visually scores the range and quality of movement during task execution. Moreover, some of these outcome assessments have been criticised for not being sensitive enough to detect clinically meaningful change in upper limb function after intervention [8]. To provide a more detailed and objective description of upper limb movement patterns, quantitative measurements are needed. Quantitative outcome measures are measurement methods that provide an objective description of the upper limb task performance based on technical measures and calculations, e.g. joint angles, movement duration, and speed. Three-dimensional (3D) movement analysis is a powerful tool for such quantitative assessment of a movement in all degrees of freedom. The 3D analysis of the lower limbs, e.g. gait analysis, has already widespread use in biomechanical research and many clinical applications. 3D analysis thus seems valuable to provide additional information on the upper limb movement patterns observed in HCP and to better understand the resulting compensations.

Nonetheless, the transfer of knowledge and experience gained from gait analysis to the upper limb turns out to be difficult. The lack of cyclic movements, the variety of functions and abundant degrees of freedom make the upper limb analysis considerably more complex [9]. To promote the standardisation of 3D upper limb movement analysis, the International Society of Biomechanics (ISB) recently published some recommendations on the definition of joint coordinate systems and rotation sequences for the trunk, shoulder, elbow, wrist and hand [10]. These recommendations are based on the ISB standard for reporting kinematic data published by Wu and Cavanagh [11]. A definition for the local axis system in each articulating segment or bone is provided for each joint, while respecting the upper limb anatomy and clinical interpretation of joint movements [10]. Upper limb joint coordinate systems and rotation orders are chosen to minimise the possible occurrence of singularity problems. For the description of shoulder rotations, this rotation order implies gimbal lock at $0^{\circ}$ and $180^{\circ}$ of elevation. If a movement around these positions is of interest, other rotation sequences are recommended, e.g. the sequence flexion-abduction-rotation is recommended for movements in the sagittal plane, and abduction-flexion-rotation for movements in the frontal plane [12]. However, a consensus on the entire protocol for 3D upper limb movement analysis is still required in order to facilitate the comparison of results and encourage communication among researchers and clinicians.

In the past decade, several studies have been published on the quantification of upper limb movements in HCP. The aim of this review was to give a comprehensive summary of results found in literature on objective measurements of upper limb movements in HCP. Clinically relevant results on spatiotemporal and/or joint angle movement characteristics will be discussed. To promote further standardisation of the protocol for 3D upper limb movement analysis in HCP, some methodological reflections will also be put forward.

\section{Literature search}

Papers were selected from following electronic databases: PubMed, CINAHL, Cochrane Library and Web of Science (until December 2007), using keywords for cerebral palsy ('cerebral palsy', 'CP') and the upper limb ('upper limb', 'upper extremity'). To limit results, these terms were combined with a search for movement patterns, including 'biomechanics or kinematics', 'movement patterns' and 'reach or grasp'. To ascertain no study was missed, an online search of journals likely to contain target articles and a manual screening of the reference lists of all included studies was conducted.

Inclusion criteria were: (1) hemiplegic cerebral palsy; (2) objective description of upper limb movement characteristics (spatiotemporal and/or joint angles); (3) full papers. Studies only describing a biomechanical upper limb model or measurement procedure without results on movement characteristics or studies primarily assessing anticipatory movement planning and motor control (feed forward and feedback control) were not considered. Studies were also excluded if they were not published in English. Two independent reviewers screened title and abstract of the selected papers for inclusion. In case of disagreement, the full article was read and discussed until consensus was reached.

The database search identified 96 articles; another eight articles were retrieved from the online journal search and reference screening. Based on the predefined inclusion and exclusion criteria, 17 articles were selected for inclusion in the current review. Study details are summarised in Tables 1-4.

Table 1

Study population.

\begin{tabular}{|c|c|c|c|c|c|}
\hline Study & Subjects & Age range & Upper limb impairment & Brain lesion & Clinical assessment \\
\hline Coluccini et al. [13] & HCP spastic $(n=5)$ & - & Mild-moderate & PVL & Melbourne Assessment \\
\hline Rönnqvist and Rösblad [17] & $\mathrm{HCP}(n=11)$ & $5-12$ & Mild-moderate & - & - \\
\hline Kreulen et al. [18] & $\mathrm{HCP}(n=10)$ & $11-27$ & - & - & AROM \\
\hline Mackey et al. [19] & HCP spastic $(n=10)$ & $10-17$ & - & - & - \\
\hline Wright et al. [14] & HCP spastic/dystonic $(n=8)$ & 4-9 & - & - & - \\
\hline Wright et al. [15] & HCP spastic/dystonic $(n=8)$ & 4-9 & - & - & - \\
\hline $\begin{array}{l}\text { Steenbergen and } \\
\text { Meulenbroek [20] }\end{array}$ & $\operatorname{HCP}(n=5)$ & $14-18$ & - & - & $\begin{array}{l}\text { Purdue Pegboard-Box\&Block } \\
\text { test-ASS }\end{array}$ \\
\hline van der Heide et al. [16] & HCP preterm $(n=34)$ & $2-11$ & Mild-moderate-severe & PVL (mild-severe) & ASS-PEDI \\
\hline Ricken et al. [23] & HCP spastic $(n=10)$ & $5-11$ & - & - & - \\
\hline Volman et al. [24] & HCP spastic $(n=12)$ & $8-14$ & Mild-moderate & - & - \\
\hline van Thiel and Steenbergen [22] & $\mathrm{HCP}(n=8)$ & $15-20$ & Mild-moderate & - & - \\
\hline Steenbergen et al. [21] & HCP spastic $(n=6)$ & $14-19$ & - & - & - \\
\hline Fitoussi et al. [26] & $\mathrm{HCP}(n=15)$ & $7-15$ & - & - & $\begin{array}{l}\text { A/PROM-ASS-sensibility-Zancolli- } \\
\text { Corry classif }\end{array}$ \\
\hline Mackey et al. [25] & HCP spastic $(n=10)$ & $5-15$ & - & - & - \\
\hline Kreulen et al. [28] & $\mathrm{HCP}(n=10)$ & $11-27$ & - & - & AROM \\
\hline Kreulen et al. [27] & $\mathrm{HCP}(n=10)$ & $5-29$ & - & - & AROM \\
\hline Hurvitz et al. [29] & HCP spastic $(n=9)$ & $7-16$ & - & - & $\begin{array}{l}\text { A/PROM-MAS-PEDI-FIM-Purdue } \\
\text { Pegboard-BOMPT }\end{array}$ \\
\hline
\end{tabular}

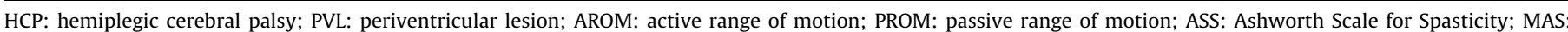

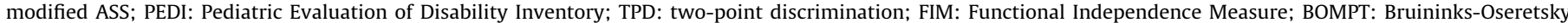
Motor Proficiency Test.

-, not reported. 
Table 2

\begin{tabular}{|c|c|c|c|c|c|c|c|c|}
\hline & \multirow[t]{2}{*}{ Reach to touch } & \multicolumn{2}{|c|}{ Reach to grasp } & \multirow[t]{2}{*}{ Grasping } & \multirow[t]{2}{*}{ Other } & \multirow[t]{2}{*}{ Condition } & \multirow[t]{2}{*}{ Start position ${ }^{\mathrm{a}}$} & \multirow[t]{2}{*}{ Reach distance $^{b}$} \\
\hline & & Spherical & Cylindrical & & & & & \\
\hline \multicolumn{9}{|l|}{ Movement patterns ${ }^{c}$} \\
\hline Coluccini et al. [13] & & $\Phi \mathrm{x}$ & & & & Self-selected speed/fast as possible & Subject specific & Arm length \\
\hline Rönnqvist and Rösblad [17] & & $\mathrm{x}$ & & $\mathrm{x}$ & & 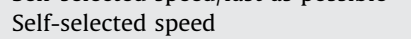 & Fixed & Fixed \\
\hline Kreulen et al. [18] & & $\Phi$ & $\Phi$ & & & Self-selected speed & Subject specific & Arm length \\
\hline Mackey et al. [19] & $\Phi \mathrm{x}$ & & & & $\Phi \mathrm{x}$ & Self-selected speed & Subject specific & Arm length \\
\hline Wright et al. [14] & & & & & $\mathrm{x}$ & Self-selected speed & Fixed & Fixed \\
\hline Wright et al. [15] & & & & & $\mathrm{x}$ & Self-selected speed & Fixed & Fixed \\
\hline Steenbergen and Meulenbroek [20] & & $\Phi \mathrm{x}$ & & & & Self-selected speed & Subject specific & Arm length \\
\hline van der Heide et al. [16] & & & $\mathrm{x}$ & & & Self-selected speed & Subject specific & Arm length \\
\hline \multicolumn{9}{|l|}{ Contextual influences } \\
\hline Ricken et al. [23] & & $\Phi \mathrm{x}$ & & & & Self-selected speed/externally paced & Fixed & Fixed \\
\hline Volman et al. [24] & $\mathrm{x}$ & & & & & Fast and accurate as possible & Fixed & Fixed \\
\hline van Thiel and Steenbergen [22] & $\mathrm{x}$ & $\mathrm{x}$ & & & $\mathrm{x}$ & Fast as possible & Fixed & Fixed \\
\hline Steenbergen et al. [21] & $\Phi \mathrm{x}$ & $\Phi \mathrm{x}$ & & $\Phi \mathrm{x}$ & & Fast and accurate as possible & Fixed & Fixed \\
\hline \multicolumn{9}{|l|}{ Repeatability } \\
\hline Fitoussi et al. [26] & & & $\Phi$ & & $\Phi$ & Self-selected speed & Subject specific & Arm length \\
\hline Mackey et al. [25] & & & & & $\Phi$ & Self-selected speed & - & - \\
\hline \multicolumn{9}{|l|}{ Therapeutic interventions } \\
\hline Kreulen et al. [28] & & $\Phi$ & & & & Self-selected speed & Subject specific & Arm length \\
\hline Kreulen et al. [27] & & & & & $\Phi$ & Self-selected speed & Subject specific & Arm length \\
\hline Hurvitz et al. [29] & $\Phi \mathrm{x}$ & & & & & Fast and accurate as possible & - & - \\
\hline
\end{tabular}

Joint kinematics $\Phi$; spatiotemporal characteristics $\mathrm{x}$; -, information was not specified.

a 'Subject specific' start position indicates an adjustment of foot and back support to the child's body height.

b 'Fixed' reaching distance indicates distances (e.g. $30 \mathrm{~cm}$ or $40 \mathrm{~cm}$ ) chosen based on the child's body height.

c Movement characteristics were compared to healthy controls. 
Table 3

Biomechanical model and movement characteristics.

\begin{tabular}{|c|c|c|c|}
\hline \multirow[t]{2}{*}{ Study } & \multirow[t]{2}{*}{ Biomechanical model } & \multicolumn{2}{|l|}{ Anatomical level } \\
\hline & & Spatiotemporal & Angular \\
\hline \multicolumn{4}{|l|}{ Movement patterns } \\
\hline Coluccini et al. [13] & Five segments ${ }^{a}$ & Wrist & Head-trunk-shoulder-elbow-wrist \\
\hline Rönnqvist and Rösblad [17] & - & Wrist-thumb-index & - \\
\hline Kreulen et al. [18] & Three segments ${ }^{\mathrm{a}}$ & - & Trunk-shoulder-elbow \\
\hline Mackey et al. [19] & Nine segments (bilateral) ${ }^{\mathrm{a}}$ & Shoulder-elbow & Trunk-shoulder-elbow \\
\hline Wright et al. [14] & - & Wrist-object & - \\
\hline Wright et al. [15] & - & Wrist & - \\
\hline Steenbergen and Meulenbroek [20] & Five segments (bilateral) & Wrist & Trunk-shoulder-elbow \\
\hline van der Heide et al. [16] & - & Wrist & - \\
\hline \multicolumn{4}{|l|}{ Contextual influences } \\
\hline Ricken et al. [23] & Five segments (bilateral) & Wrist & Trunk-shoulder-elbow \\
\hline Volman et al. [24] & - & Pen signals & - \\
\hline van Thiel and Steenbergen [22] & - & Shoulder-wrist & - \\
\hline Steenbergen et al. [21] & Five segments (bilateral) & Wrist & Trunk-shoulder-elbow \\
\hline \multicolumn{4}{|l|}{ Repeatability } \\
\hline Fitoussi et al. [26] & Four segments ${ }^{\mathrm{a}}$ & - & Shoulder-elbow-wrist \\
\hline Mackey et al. [25] & Seven segments (bilateral) ${ }^{a}$ & - & Shoulder-elbow \\
\hline \multicolumn{4}{|l|}{ Therapeutic interventions } \\
\hline Kreulen et al. [28] & Three segments $^{\mathrm{a}}$ & - & Trunk-shoulder-elbow \\
\hline Kreulen et al. [27] & Three segments $^{\mathrm{a}}$ & - & Elbow \\
\hline Hurvitz et al. [29] & - & Elbow & Elbow \\
\hline
\end{tabular}

- , not applicable.

a Segments defined by at least three non-collinear markers per segment (rigid bodies).

\section{Table 4}

Measurement methods.

\section{Movement patterns}

Coluccini et al. [13]

Rönnqvist and Rösblad [17]

Kreulen et al. [18]

Mackey et al. [19]

Wright et al. [14]

Wright et al. [15]

Steenbergen and Meulenbroek [20]

van der Heide et al. [16]

Optoelectronic motion analysis system (BTS)

ProReflex System

2 synchronised S-VHS video cameras

MotionAnalysis video system

Single S-VHS video camera with image splitting device

Single S-VHS video camera with image splitting device

Optotrak 3020 system

ELITE-optoelectronic movement recording system

Dual CODA mpx3 motion analysis system

Wireless pen and digitizer

Optotrak 3020 system

Optotrak 3020 system

VICON optoelectronic system

MotionAnalysis video system

2 synchronised S-VHS video cameras

2 synchronised S-VHS video cameras

Electrogoniometer
Passive optical system Passive optical system Passive optical system Passive optical system Passive optical system Passive optical system Active optical system Passive optical system

Active optical system

Active optical system Active optical system

Passive optical system Passive optical system

Passive optical system Passive optical system

\section{Methodological aspects}

Table 1 gives an overview of the study populations. Sample sizes ranged from 5 to 34 subjects (median 10) and age ranged from 2 to 29 years (not reported in one study). Subject's gender, age and hemiplegic side were clearly stated in six studies. In four studies, children with dyskinetic movement disorders or diplegic $\mathrm{CP}$ were also included [13-16]. Inclusion/exclusion criteria were well defined in all but three studies $[18,21,22]$. Eight studies clinically evaluated the upper limb function and five studies graded the severity of upper limb impairment (mild, moderate or severe) based on clinical assessments or a classification system [13,16-18,22,24]. Two studies described the type of brain lesions [13,16].

The aim and study set-up differed in the 17 papers (Table 2 ). In general, two main groups can be distinguished: (1) studies describing the upper limb movement characteristics in HCP (12 studies) and (2) studies assessing the repeatability and sensitivity of the measurement procedure (five studies). Within the first group, eight studies compared the upper limb movement patterns in HCP to healthy controls [18-20] and four studies compared the performance of the hemiplegic upper limb to the non-hemiplegic side [21-24]. The latter studies also studied the influence of task context, e.g. object size and reaching distance. Within the second group, two studies assessed the repeatability of a 3D upper limb movement analysis $[25,26]$ and three studies evaluated whether the effect of therapeutic interventions on upper limb movement patterns could be objectively measured [27-29].

The majority of the studies described upper limb movements during 'reach to grasp' (10/17) or 'reach to touch' (6/17). Other tasks involved 'grasping' $(2 / 17)$ gross motor functions (e.g. hand to mouth/head, hitting) (3/17) or complex motor tasks (posting task) (2/17). Children were instructed to move at self-selected speed in all studies comparing HCP vs. controls [13-20], in one study comparing the hemiplegic with the non-hemiplegic side [23], for both repeatability studies $[25,26]$, and in two intervention studies $[27,28]$. Children were instructed to move as fast as possible in five studies $[13,21,22,24,29]$, of which three studies also imposed an accuracy demand $[21,24,29]$. External pacing was used in one study [23].

All studies provided some standardisation of the reference position: sitting in 16 studies, standing in one study [23]. Eight studies adopted foot and back support to the child's anthropometrical measures and reaching distance to the child's arm length (Table 2). 
Described movement characteristics are listed in Table 3. Twelve studies described spatiotemporal characteristics [13-17,19-24,29]: movement duration, movement speed (mean and maximum velocity, timing of maximum velocity), deceleration time, distance and straightness of the movement trajectory, smoothness and grasp aperture. Straightness of the movement trajectory was described as the ratio between the distance travelled from start to end-position (summation) and the direct, shortest distance between both positions [30] Smoothness was determined by the number of movement units within one task execution, e.g. one reach. The definition of a movement unit is based on the velocity profile of the movement, whereby each unit consists of an acceleration phase and a deceleration phase [30]. These spatiotemporal characteristics were mainly based on wrist movements, although some authors also used the elbow or shoulder $[19,22,29]$. Eleven studies calculated joint kinematics (joint angles) at the level of the trunk, shoulder, elbow or wrist in the sagittal, frontal and/or transverse plane $[13,18-21,23,25-29]$. The rigid body assumption to describe movements of the different segments involved, with at least three non-collinear markers per segment was adopted by seven authors [13,18,19,25-28], of whom five authors used skin markers during movement registration [18,19,26-28] and two authors used segment marker clusters [13,25]. Others determined joint angles based on one or two skin markers per segment by means of 3D vector calculations $[20,21,23]$, or an electrogoniometer [29]. All authors defined shoulder movements as a movement between the upper arm and the trunk. The recently published ISB recommendations concerning the definition of upper limb joint coordinate systems and joint rotation sequences [10] were not yet implemented in any of the studies.

Different tracking methods were used to study upper limb movements (Table 4): passive $(11 / 17)$ or active $(4 / 17)$ optical systems, electrogoniometric measures (1/ $17)$ and a wireless digitizing pen (1/17). The accuracy and reliability of these tracking methods have already been discussed in detail in the papers of Chiari et al and Anglin and Wyss [31,32].

\section{Results}

\subsection{Movement characteristics}

In the following section, movement characteristics will be presented for the different tasks, including spatiotemporal characteristics and joint kinematics (joint angles), as well as contextual influences. First, the comparison of the hemiplegic side (HS) in children with HCP vs. the non-dominant side in healthy controls (CON) is reported, followed by the differences between the HS and the non-hemiplegic side (NHS) in HCP and the comparison between the NHS and the dominant side in CON. All reported results indicate significant differences, unless otherwise stated. Whenever possible, exact data are also provided. Results for joint kinematics are reported as mean or median differences in joint angle degrees between children with HCP and CON, or between the HS and NHS.

\subsubsection{Reach to touch and reach to grasp}

4.1.1.1. Spatiotemporal characteristics. The differences between HCP and CON during 'reach to touch' and 'reach to grasp' were studied by Mackey et al. [19], Coluccini et al. [13] and Rönnqvist and Rösblad [17]. All authors reported longer movement durations in HCP. However, Rönnqvist and Rösblad [17] only described significant differences between children with moderate HCP and CON, and not between mild HCP and CON. For the group of moderate $\mathrm{HCP}$, they also described reduced trajectory straightness and smoothness compared to both mild HCP and CON. All children with HCP showed a later timing of maximum hand velocity with a higher velocity at object contact vs. CON for 'reach to grasp' [17] and lower maximum angular elbow velocities for 'reach to touch' [19].

Comparison of the HS vs. the NHS during both tasks showed longer movement durations and deceleration times before grasping, reduced movement smoothness and lower mean hand velocities at the HS (Steenbergen et al. [21], van Thiel and Steenbergen [22], Ricken et al. [23], Rönnqvist and Rösblad [17]). Some authors also reported a difference in timing of maximum hand and shoulder velocity between both sides, with the hand reaching its maximum velocity before the shoulder at the HS, opposite to what occurs at the NHS [21,22]. Rönnqvist and Rösblad [17] were the only authors who reported significantly lower maximum hand velocities at the HS.

Comparing the NHS in HCP vs. the dominant side in CON during 'reach to grasp' showed longer movement durations, lower mean hand velocities and a later timing of maximum hand velocity, along with reduced trajectory straightness and movement smoothness at the NHS $[13,16,17,20]$.

4.1.1.2. Joint kinematics. Mackey et al. [19] compared the differences between HCP and CON in joint angles at the elbow, shoulder and trunk during 'reach to touch'. They found a reduced elbow extension (difference of $21^{\circ}$ ) in HCP, and no significant differences in shoulder and trunk movements. Coluccini et al. [13] and Kreulen et al. [18] studied joint angles during 'reach to grasp'. Coluccini et al. [13] only presented a spherical object (requiring no forearm supination), while Kreulen et al. [18] also presented a vertically oriented cylinder (requiring forearm supination). Coluccini et al. [13] reported significantly less shoulder abduction and elbow extension, increased wrist flexion and ulnar deviation, together with increased trunk flexion and lateral flexion in HCP, though all differences were below $10^{\circ}$. Kreulen et al. [18] only observed significantly increased trunk flexion in HCP during 'reach to grasp a spherical object'. In contrast, when presenting a vertically oriented cylinder, the latter authors reported increased shoulder elevation (difference of $18^{\circ}$ ) in the sagittal plane, increased shoulder internal rotation (difference of $21^{\circ}$ ), less elbow extension (difference of $31^{\circ}$ ) and supination (difference of $45^{\circ}$ ), together with increased trunk movements (differences between $10^{\circ}$ and $14^{\circ}$ in all planes) in HCP [18].

Studies comparing the HS vs. the NHS, showed less elbow extension (differences between $9^{\circ}$ and $21^{\circ}$ ), shoulder flexion (differences between $5^{\circ}$ and $13^{\circ}$ ) and inconsistent results for shoulder elevation at the HS during both 'reach to touch' and 'reach to grasp a spherical object' [21,23]. Steenbergen et al. [21] additionally reported increased trunk flexion, but no differences in trunk rotation. In contrast, Ricken et al. [23] reported increased trunk lateral flexion (difference of $6^{\circ}$ ) and rotation (difference of $19^{\circ}$ ) when reaching with the HS.

Comparing the NHS in HCP to the dominant side in CON during 'reach to grasp' gives inconsistent results. Steenbergen and Meulenbroek [20] reported less elbow extension (difference of $10^{\circ}$ ) at the NHS, but no differences in shoulder range of motion or trunk flexion. Coluccini et al. [13] did find a reduced shoulder abduction and flexion and increased wrist flexion and ulnar deviation at the NHS, though all differences were below $10^{\circ}$.

4.1.1.3. Task context. Coluccini et al. [13] and Ricken et al. [23] evaluated the effect of movement speed and external pacing, respectively, during 'reach to grasp'. Executing the task as fast as possible vs. self-selected speed did not reduce the differences in spatiotemporal characteristics and joint kinematics between HCP and CON [13]. Externally pacing the movement by presenting a moving object to be grasped, only resulted in a shorter deceleration time at the HS [23].

The effect of object size and task functionality on spatiotemporal characteristics of the HS and NHS was evaluated by van Thiel and Steenbergen [22] and Volman et al. [24], respectively. Reaching for smaller objects did not influence the movement duration or smoothness at the HS [22]. Task functionality was modulated in a 'reach to touch' task by presenting a non-functional switch, a switch that could be pressed (semi-functional) or a light switch (functional). Increasing task functionality resulted in reduced movement durations and smoother movements at both the HS and NHS, and more symmetric, bell-shaped velocity profiles (more normal) at the HS. Functionality of the task did not influence 
the maximum hand velocity, or timing of maximum hand velocity at either side [24].

Steenbergen and Meulenbroek [20] examined the effect of reaching distance on spatiotemporal characteristics and joint angles, at the NHS compared to the dominant side in CON. They found increased movement durations, higher maximum hand velocities and an earlier timing of maximum hand velocity with increasing reach distance. 'Reach to grasp' an object beyond arm length also resulted in increased elbow extension and trunk flexion, reducing the difference between the NHS and the dominant side in $\mathrm{CON}$.

\subsubsection{Grasping}

4.1.2.1. Spatiotemporal characteristics. Comparing HCP vs. CON, Coluccini et al. [13] described longer movement durations to grasp and release an object in HCP and Rönnqvist and Rösblad [17] described a later timing of maximum grip aperture, measured as the linear space between thumb and index finger, in children with moderate HCP compared to both mild HCP and CON. Children with moderate HCP also used a whole hand, palmar-like grasp, without anticipatory grasp formation. The group of mild HCP showed a larger maximum grip aperture compared to $\mathrm{CON}$, but no significant differences in grip aperture were seen at object contact.

Comparing the HS vs. the NHS, Steenbergen et al. [21] and Rönnqvist and Rösblad [17] reported a longer time for proper positioning of the hemiplegic hand for grasping. While Steenbergen et al. [21] reported lower velocities at object contact, Rönnqvist and Rösblad [17] observed a higher hand velocity at object contact at the HS (mild and moderate HCP).

\subsubsection{Joint kinematics. Not performed.}

\subsubsection{Task context. Not described.}

\subsubsection{Other motor functions}

Mackey et al. [19] studied the differences between HCP and CON during 'hand to mouth/head'. Wright et al. [14,15] used a more complex motor task of 'object posting' (putting objects of different shapes into holes with matching contours) to study the differences between both groups. The performance of the HS vs. the NHS was compared during a 'hitting' task, where children were instructed to hit a projected target with a hand-held rod (van Thiel and Steenbergen [22]).

4.1.3.1. Spatiotemporal characteristics. Longer movement durations were seen in HCP compared to CON for all tasks $[14,15,19]$. During 'hand to mouth', children with HCP also had lower maximum elbow angular velocities compared to CON, but this significant difference was not seen during 'hand to head'. For both tasks, no differences in timing of maximum angular elbow velocity were observed [19]. When manipulating different objects for the 'object posting' task [14,15], children with HCP moved with lower mean velocities, they generated more errors during reaching, made more adjustment errors (angular and rotational adjustments) and displayed poor adjustment efficiency [14,15].

Comparing the HS vs. the NHS during 'hitting' only showed longer movement durations at the HS. No significant differences were found between both sides for movement smoothness or timing of maximum shoulder and hand velocities [22].

4.1.3.2. Joint kinematics. Comparing children with HCP vs. CON, Mackey et al. [19] reported no differences in elbow extension, but significantly less forearm supination (differences between $26^{\circ}$ and $28^{\circ}$ ) and increased trunk flexion (difference of $14^{\circ}$ ) during 'hand to mouth/head' in HCP. They also found increased shoulder elevation (difference of $21^{\circ}$ ) during 'hand to mouth', which was not observed during 'hand to head'.

Comparison of the NHS in HCP vs. the dominant side in CON, showed no significant differences in joint angles at the shoulder, elbow and trunk [19].

4.1.3.3. Task context. The influence of object size and shape on spatiotemporal characteristics during 'hitting' and 'object posting', respectively, was investigated by van Thiel and Steenbergen [22] and Wright et al. [14,15]. Hitting a smaller object, thus increasing accuracy demands, resulted in reduced movement smoothness at both sides [22]. Object shape (cone vs. cylinder vs. triangle) affected the difficulty level of the posting task. Increasing task difficulty required more adjustment movements to successfully post the object, and resulted in more adjustment inefficiency in HCP. Children with HCP were not able to post the triangle (as opposed to the cone and cylinder), whereas this object did not cause any problems in CON $[14,15]$.

The effect of task repetition was reported for the 'posting task', with more repetitions resulting in reduced movement durations, improved adjustment efficiency and higher mean velocities compared to the initials trials in the group of HCP $[14,15]$.

\subsection{Repeatability and sensitivity of the measurement procedure}

\subsubsection{Repeatability}

Mackey et al. [25] and Fitoussi et al. [26] assessed within and between session repeatability of the joint angle patterns (waveforms) using the coefficient of multiple correlation (CMC) [33] in 10 and 15 hemiplegic children, respectively. Mackey et al. [25] evaluated shoulder and elbow waveforms during 'hand to mouth/ head'. They reported good to excellent levels of within and between session repeatability, for both tasks, for shoulder and elbow sagittal plane kinematics (CMC 0.87-0.96) and moderate to good levels of within and between session repeatability for frontal and transverse kinematics (CMC 0.62-0.90). Shoulder rotations had lowest values of repeatability for both tasks, especially between sessions (CMC 0.49-0.63). Fitoussi et al. [26] evaluated shoulder, elbow and wrist waveforms during 'reach to grasp' and 'hand to mouth'. They reported good to excellent within session repeatability for both tasks, for all joint movements $(\mathrm{CMC}>0.84)$, with the exception of wrist sagittal plane kinematics (CMC-values not reported). Between session repeatability was excellent for both tasks for all joint movements (CMC > 0.84), except for shoulder rotations during 'hand to mouth' (CMC 0.82) and wrist deviations during both tasks (CMC 0.68-0.78). Both authors reported more variation in joint angles at start position and the first $20 \%$ of the waveforms, within and between sessions, although exact data were not provided.

\subsubsection{Sensitivity to change}

Kreulen et al. $[27,28]$ evaluated the effect of surgical correction of the pronation deficit. They found that this deficit induced excessive trunk lateral flexion and rotation, increased humeral internal rotation and elbow flexion during activities requiring forearm supination (reach to grasp a drinking glass). These abnormal movements reduced or disappeared postoperatively, with a significant increase of the maximum active supination and elbow extension (mean increase $46^{\circ}$ and $13^{\circ}$, respectively) and a significant reduction of humeral internal rotation and trunk lateral flexion (mean decrease $25^{\circ}$ and $9^{\circ}$, respectively). However, maximum active pronation also reduced postoperatively (mean loss $17^{\circ}$ ). Activities requiring active forearm pronation (reach to grasp wooden disc) were executed with less pronation postoperatively, less humeral internal rotation (mean decrease $17^{\circ}$ ) and increased elbow extension (mean increase $13^{\circ}$ ). Due to the 
pronation loss, new compensatory movements arose, e.g. increased humeral abduction and trunk lateral flexion contralateral.

Hurvitz et al. [29] assessed the effect Botulinum toxin type-A (BTX-A) injections on the elbow extension pattern during a 'reach to touch' task, using an electrogoniometer. They reported reduced movement durations, improved trajectory straightness and more symmetrical velocity profiles at the elbow, with no initial elbow flexion before elbow extension at 18 weeks post-BTX. No sustaining significant effects were reported on range of motion, movement duration or trajectory after 24 weeks.

\section{Discussion}

The current review was set out to give a comprehensive summary of results found in literature on objective measurements of upper limb movement characteristics in HCP. Studies showed discernible differences in marker configurations and segments, analysed tasks, study set-up, and outcome measures. These diverse methodological approaches make the comparison of published results challenging and complicate the reporting of objective data when summarising results. Despite this consideration, several conclusions can be made about upper limb movement characteristics in HCP.

Different studies have shown longer movement durations, reduced movement speed, smoothness and trajectory straightness of the hand at the HS when executing upper limb tasks at selfselected speed [13-15,17,19]. According to the definition of von Hofsten, smoothness can be determined by the number of movement units within one task execution [30]. The definition of a movement unit is based on the velocity profile of the movement, whereby each unit consists of an acceleration phase and a deceleration phase [30]. Following authors reported the number of movement units based on the above stated definition: Rönnqvist and Rösblad [17] and van der Heide et al. [16]. Other authors stated that a movement unit was determined as one acceleration followed by one subsequent deceleration (Volman et al. [24]), as the number of peaks and valleys (van Thiel and Steenbergen [22]), or as the number of zero crossings (Steenbergen et al. [21], Wright et al. [14]). All studies used the tangential velocity or acceleration profile of the hand/wrist to determine the number of movement units. The observed movement slowness is more pronounced for grasping than for reaching, indicating more distal movement control problems [17,21]. Moving as fast as possible did not result in a normalisation of the spatiotemporal characteristics at the HS, though this was evaluated in only one study [13]. Increasing task functionality, on the other hand, improved movement duration and smoothness at the HS [22,24], while increased reaching distance improved movement duration and speed at the NHS [20]. The spatiotemporal characteristics described in HCP are similar to the immature reaching patterns seen in very young children. The first reaching movements, observed at the age of 3-4 months, are characterised by considerably variable and irregular hand movement trajectories, with predominant trunk involvement to bring the hand closer to the object [34,35]. Infant reaching shows remarkable improvement of the hand trajectories over the first 2 years of life, with proximodistal maturation [36]. Further maturation towards adult multi-joint coordination, with smooth and stable hand trajectories and minimal trunk involvement continues until the age of 8-10 years [37]. It appears that children with HCP never attain these adult-like reaching patterns. The change in spatiotemporal reaching characteristics in $\mathrm{HCP}$ with increasing age was described in one study, though only at the NHS [16]. Children with mild to moderate impairment showed an improved reaching pattern (movement speed and trajectory straightness), whereas severely disabled children moved slower and less straight with increasing age [16]. Further study of the upper limb spatiotemporal characteristics at the HS could help to improve our understanding of the maturation of the pathological movement patterns in HCP.

While spatiotemporal characteristics have been extensively reported, merely six studies compared upper limb joint kinematics at the HS to either the NHS or CON $[13,18-21,23]$. These studies confirm our clinical impression of children with HCP using less elbow extension and supination $[13,18,19]$, and compensatory trunk movements (mainly trunk flexion) to bring the hand closer to the object. These compensatory trunk movements were also seen during hand to head/mouth [19]. All six studies reported trunk, shoulder and/or elbow joint movements, whereas joint movements of the wrist were reported in only one study [13]. The maturation of angular reaching patterns has only been described in typically developing children (Petuskey et al. [38]). They established normal paediatric upper limb joint kinematics during different tasks in a group of 51 typically developing children (age 5-18 years). These authors reported significant age differences in joint kinematics at the level of the head, trunk, elbow and wrist, for each task, though differences were below $10^{\circ}$.

Even though it is known that children with HCP present with a variety of upper limb impairments and a diversity of affected brain structures, studies included in this review made little distinction within the group of hemiplegic children. Sample sizes in the different studies were small and thus a further division into subgroups would be less viable for statistical comparison. Still, evaluating these children as one homogeneous group will undoubtedly result in information loss on specific movement characteristics. No more than five studies classified the severity of upper limb impairment (mild, moderate, severe), of which only van der Heide et al. [16] and Rönnqvist and Rösblad [17] determined the relation between this classification and the spatiotemporal characteristics of the movement patterns. The other studies merely used the classification to describe the study group. Moreover, there was no equivalence between the classification systems used in the different studies. The discrepancies in the choice of a classification system to grade the severity of upper limb impairment and the relevance of using these classification systems, remains a challenge for future research. Rönnqvist and Rösblad [17] compared the HS between children with mild vs. moderate upper limb impairment and found that children with mild impairment only experienced problems with fine distal arm and hand movements. van der Heide et al. [16] compared the NHS between children with mild, moderate and severe upper limb impairment and reported lower maximum hand velocities and reduced trajectory straightness at the NHS with increasing impairment at the HS. Recent studies have also suggested that the type and location of the brain lesion distinguish children with HCP regarding motor and sensory impairments and upper limb abilities [39-41]. van der Heide et al. [16] related the severity of periventricular lesions to the spatiotemporal characteristics of the NHS and reported reduced smoothness and trajectory straightness at the NHS in children with more severe brain lesions.

To increase our understanding of the pathological movement patterns and compensatory movements seen in HCP, future studies will need to address all involved upper limb joints at the HS (in all three movement planes), along with the changes in joint angular patterns with increasing age. Relating the upper limb movement characteristics to the upper limb impairments and the type of brain lesion will also add to our insights in the underlying mechanisms of the pathological movements. This gained knowledge could then help in the construction of a classification scheme to characterise the pathological upper limb movement patterns in children with HCP. However, as the development and clinical implementation of 3D upper limb movement analysis is still at early stages, the design 
of any such classification scheme will be a next step for future research. Since all studies describing the NHS indicated significant differences in spatiotemporal characteristics and joint kinematics compared to healthy controls during upper limb task execution, the NHS cannot be regarded as 'normal'. It is therefore recommended to use a group of healthy controls when studying the pathological upper limb movement patterns in HCP. Coluccini et al. [13] and Petuskey et al. [38] made a first step in the construction of proper normal values for children. However, both studies did not comply with the recently published ISB recommendations. It seems that only van Andel et al. [42] have established normal values conform the ISB recommendations, though only for adults.

Gait analysis (lower limb 3D movement analysis) is currently perceived as the golden standard for decision making in lower limb surgery, further treatment planning and follow-up. To allow an analogous process of clinical decision making for the upper limb, results from the $3 \mathrm{D}$ upper limb movement analysis need to be converted into useful information at the level of the structures that are treated by the surgeon (muscles, bones). Given the complex anatomy of the upper limb, the variety of functions and non-cyclic, free nature of upper limb movements, this conversion will not be straightforward and care should be taken in the transfer of knowledge from gait analysis to 3D upper limb movement analysis. Moreover, to allow clinical decision making based on 3D upper limb movement analysis or to evaluate treatment effects, the measurement procedure has to be proven reliable, sensitive to change and applicable in HCP. To the best of our knowledge, to date only two studies assessed the repeatability of such a 3D upper limb movement analysis in children with HCP $[25,26]$. The repeatability of joint angle patterns seemed promising [25,26], though methodological differences must be considered when interpreting results (e.g. definition of the biomechanical model, selected tasks and techniques to delimit movement cycles). Moreover, both authors only reported CMC-values, which might be insufficient to establish the reliability of an entire measurement procedure. Other factors that could influence the outcome should also be taken into account, e.g. soft-tissue artifacts, marker placement and demarcation of movement cycles. On the other hand, these studies emphasised the importance of a thorough standardisation of the study set-up to ensure maximum repeatability of the task performance, e.g. adopting reaching distance and height to the anthropometric measures of the child (arm length and shoulder height), together with the foot and back support and the initial position for every task. As increased trunk involvement was reported in most studies included in this review, the importance of unrestricted task execution has been highlighted.

Three studies used 3D movement analysis to assess upper limb treatment effects [27-29]. One study evaluated the effect of BTX-A injections with an electrogoniometer and reported a short term effect on the elbow extension pattern during reaching [29]. Evaluation of the effect of surgical correction of the pronation deficit not only indicated increased maximum elbow supination, but also demonstrated a significant change in upper limb joint kinematics at the level of the trunk, shoulder and elbow after treatment $[27,28]$. Although these studies limited the analysis to end-positions only, without movement registration of the upper limb from all sides, 3D upper limb movement analysis seems promising to detect changes after intervention in children with HCP. The value of 3D upper limb movement analysis to evaluate treatment effects has already been demonstrated in children with brachial plexus palsy $[43,44]$ and stroke patients $[45,46]$.

Apart from the reliability and sensitivity of a measurement procedure, its applicability (child friendly, test duration and analysis) and the relevance of upper limb tasks are also very important aspects to allow clinical implementation. Since there is no single most important upper limb function, standard activities covering important upper limb functions must be determined and defined in a movement protocol. Tasks should be functional and clinically relevant to represent a wide range of ADL. Selection is preferably based not only on literature, but also on discussions with experts in the field of HCP and tasks assessed in standardised clinical scales, e.g. the Melbourne Assessment [5], or the SHUEE [7].

The diversity in methodological approaches used in the different studies demonstrates the lack of a general consensus in literature regarding the protocol for 3D upper limb movement analysis. The need for standardisation of the protocol for 3D upper limb movement analysis has already been put forward by Rab et al. [47]. Such a protocol not only defines the biomechanical model, but also describes some standardised procedures for data collection, processing, analysis and reporting of results [48]. Despite the fact that the ISB recently published some recommendations on the biomechanical model [9], and Kontaxis et al. [48] additionally proposed a flexible framework to support the definition of standard protocols, these standardisation efforts have not yet been put into practice to study upper limb movement characteristics in HCP.

As for gait analysis, the ISB committee recommends to use marker clusters fixed at the segmental centre of mass [10] and to calibrate bony landmarks with reference to these segmental clusters (CAST-method [49]) as using skin markers tends to be erroneous due to possible soft-tissue artifacts [50]. Since these soft-tissue artifacts are considerably more pronounced during upper limb movements compared to lower limb movements, especially for the humerus and the scapula, the use of the CASTmethod is further justified. However, most authors placed markers over bony landmarks to calculate joint angles [18-21,23,25-28]. The ISB also recommends distinct joint rotation sequences for the different joints involved in the upper limb, since the clinical and easy-to-understand rotation sequence of forward flexion, abduction and rotation is less appropriate when reporting glenohumeral and scapular movements [51,52]. Recent studies have even suggested using a task-specific rotation sequence for the shoulder $[12,48,52,53]$. Nonetheless, all authors in this review used the sequence of flexion-abduction-rotation for all joints, and all tasks. Kreulen et al. $[18,27]$ were the only authors who used the globe method to define shoulder movements, which has proven to be identical to the ISB recommendations for the shoulder [54]. The biomechanical shoulder models used in the studies restricted shoulder movements to a movement between the humerus and the trunk, omitting the scapula [13,18-21,23,25-28]. Such simplification results in information loss on the coordinated interaction of all joints involved in the shoulder complex, which is necessary to better understand the abnormal movement patterns. However, the scarce knowledge in this area of upper limb 3D movement analysis can only be augmented by means of future research. The role of the scapula in the normal movement of the humerus has been well established [55]. Therefore, scapular movement should be taken into account. Whether it is clinically relevant to decompose the movement between the trunk, scapula and humerus into scapular-humeral or scapular-thoracic movements to better understand the abnormal movements, needs further exploration. A first step in this process has been undertaken by Garofalo et al. [56]. These authors proposed a new protocol to measure coordinated movements of the shoulder-girdle and the humerus in clinical settings, including scapular-thoracic movements, humerus-trunk movements and shoulder-girdle movements. One other important aspect regarding the shoulder model entails the calculation of the shoulder joint centre. Four techniques have been described in literature to calculate the shoulder joint rotation centre: regression analysis [57], calculation of the pivot point of instantaneous helical axes (IHA) [58], sphere fitting [58] or 
as an offset from external landmark positions [59]. The ISB committee recommends the IHA-method, since it is the most reliable and accurate [58]. Studies in the current review considered the shoulder joint rotation centre as an offset from external markers $[13,18,19,25-28]$ or even as an external marker itself $[20,21,23]$.

The methodological differences are expected to produce inconsistent results and as such impact on clinical interpretation. It is therefore imperative that authors use the same set of bony landmarks, joint coordinate systems and rotation sequences as proposed by the ISB committee. Such standardisation of joint movements necessitates the development of a 3D upper limb movement analysis, which in turn is indispensable for accurate upper limb evaluation and at long last will also facilitate treatment planning.

\section{Conclusion}

Several studies were published on objective measurements of upper limb movements in HCP, all using different marker configurations, study set-ups, upper limb tasks, and outcome measures. This review emphasised the need for a standardisation of the protocol for 3D upper limb movement analysis, including the ISB recommendations. Moreover, a movement protocol with functional and clinically relevant upper limb tasks has to be defined before such measurement procedure can be routinely used as an evaluation tool in children with HCP. The authors believe that future research, based on larger patient groups and taking into account the various degrees of upper limb impairment and diversity of affected brain structures in HCP, will improve our understanding of the pathological upper limb movements and compensatory movements observed in these children. These insights are indispensable for future adequate treatment planning.

\section{Acknowledgements}

Ellen Jaspers received a $\mathrm{PhD}$ fellowship of the Research Foundation-Flanders (FWO), Belgium.

\section{Conflict of interest}

The authors report no conflict of interest.

\section{References}

[1] Himmelmann K, Hagberg G, Beckung E, Hagberg B, Uvebrant P. The changing panorama of cerebral palsy in Sweden. IX. Prevalence and origin in the birthyear period 1995-1998. Acta Paediatr 2005;94(3):287-94.

[2] Uvebrant P. Hemiplegic cerebral palsy. Aetiology and outcome. Acta Paediatr Scand Suppl 1988;345:1-100.

[3] Cooper J, Majnemer A, Rosenblatt B, Birnbaum R. The determination of sensory deficits in children with hemiplegic cerebral palsy. J Child Neurol 1995;10(4):300-9.

[4] Walsh G, Brown K. Hand function in hemiplegia. In: Neville B, Goodmans R, editors. Congenital hemiplegia. London: Mac Keith Press; 2000.

[5] Randall M, Johnson L, Reddihough D. The Melbourne Assessment of Unilateral Upper Limb Function: test administration manual. Melbourne, Australia: Arena Printing; 1999.

[6] DeMatteo C, Law M, Russell D, Pollock N, Rosenbaum P, Walters S. Quality of Upper Extremity Skill Test. Ontario: Neurodevelopmental Clinical Research Unit; 1992.

[7] Davids JR, Peace LC, Wagner LV, Gidewall MA, Blackhurst DW, Roberson WM Validation of the Shriners Hospital for Children Upper Extremity Evaluation (SHUEE) for children with hemiplegic cerebral palsy. Bone Joint Surg Am 2006;88(2):326-33.

[8] Sätilä H, Kotamäki A, Autto-Rämö I. Upper limb function after botulinum toxin A treatment in cerebral palsy: two years follow-up of six cases. Pediatr Rehabil 2006;9(3):247-58.

[9] Rau G, Disselhorst-Klug C, Schmidt R. Movement biomechanics goes upwards: from the leg to the arm. J Biomech 2000;33(10):1207-16.

[10] Wu G, van der Helm FCT, Veeger HEJ, Makhsous M, Van Roy P, Anglin C, et al. ISB recommendation on definitions of joint coordinate systems of various joints for the reporting of human joint motion. Part II. Shoulder, elbow, wrist and hand. J Biomech 2005;38(5):981-92.

[11] Wu G, Cavanagh PR. ISB recommendations for standardization in the reporting of kinematic data. J Biomech 1995;28(10):1257-61.

[12] Cutti AG, Giovanardi A, Rocchi L, Davalli A, Sacchetti R. Ambulatory measurement of shoulder and elbow kinematics through inertial and magnetic sensors. Med Biol Eng Comput 2008;46(2):169-78

[13] Coluccini M, Maini ES, Martelloni C, Sgandurra G, Cioni G. Kinematic characterization of functional reach to grasp in normal and in motor disabled children. Gait Posture 2007;25(4):493-501.

[14] Wright MG, Hunt LP, Stanley $\mathrm{OH}$. Object/wrist movements during manipulation in children with cerebral palsy. Pediatr Rehabil 2005;8(4): 263-71.

[15] Wright MG, Hunt LP, Stanley OH. Quantification of object manipulation in children with cerebral palsy. Pediatr Rehabil 2001;4(4):187-95.

[16] van der Heide JC, Fock JM, Otten B, Stremmelaar E, Hadders-Algra M. Kinematic characteristics of reaching movements in preterm children with cerebral palsy. Pediatr Res 2005;57(6):883-9.

[17] Rönnqvist L, Rösblad B. Kinematic analysis of unimanual reaching and grasping movements in children with hemiplegic cerebral palsy. Clin Biomech (Bristol Avon) 2006;22(2):165-75

[18] Kreulen M, Smeulders MJC, Veeger HEJ, Hage JJ. Movement patterns of the upper extremity and trunk associated with impaired forearm rotation in patients with hemiplegic cerebral palsy compared to healthy controls. Gait Posture 2007;25(3):485-92.

[19] Mackey AH, Walt SE, Stott NS. Deficits in upper-limb task performance in children with hemiplegic cerebral palsy as defined by 3-dimensional kinematics. Arch Phys Med Rehabil 2006;87(2):207-15.

[20] Steenbergen B, Meulenbroek R. Deviations in upper-limb function of the lessaffected side in congenital hemiparesis. Neuropsychologia 2006;44(12): 2296-307.

[21] Steenbergen B, van Thiel E, Hulstijn W, Meulenbroek RGJ. The coordination of reaching and grasping in spastic hemiparesis. Hum Mov Sci 2000;19(1):75105

[22] van Thiel E, Steenbergen B. Shoulder and hand displacements during hitting, reaching and grasping movements in hemiparetic cerebral palsy. Motor Control 2001;5(2):166-82.

[23] Ricken AXC, Bennet SJ, Savelsbergh GJP. Coordination of reaching in children with spastic hemiparetic cerebral palsy under different task demands. Motor Control 2005;9(4):357-71.

[24] Volman MJM, Wijnroks A, Vermeer A. Effect of task context on reaching performance in children with spastic hemiparesis. Clin Rehabil 2002;16(6): 684-92.

[25] Mackey AH, Walt SE, Lobb GA, Stott NS. Reliability of upper and lower limb three-dimensional kinematics in children with hemiplegia. Gait Posture $2005 ; 22(1): 1-9$.

[26] Fitoussi F, Diop A, Maurel N, Laassel EM, Pennecot GF. Kinematic analysis of the upper limb: a useful tool in children with cerebral palsy. J Pediatr Orthop B 2006;15(4):247-56.

[27] Kreulen M, Smeulders JC, Veeger HEJ, Hage JJ, Van der Horst MAM. Threedimensional video analysis of forearm rotation before and after combined pronator teres rerouting and flexor carpi ulnaris tendon transfer surgery in patients with cerebral palsy. J Hand Surg Br 2004;29(1):55-60.

[28] Kreulen M, Smeulders MJC, Veeger HEJ, Hage JJ. Movement patterns of the upper extremity and trunk before and after corrective surgery of impaired forearm rotation in patients with cerebral palsy. Dev Med Child Neurol 2006;48(6):436-41.

[29] Hurvitz EA Conti GE Brown SH. Changes in movement characteristics of the spastic upper extremity after botulinum toxin injection. Arch Phys Med Rehabil 2003;84(3):444-54.

[30] von Hofsten C. Development of visually directed reaching: the approach phase. J Hum Mov Stud 1979;5:160-78.

[31] Chiari L, Della Croce U, Leardini A, Cappozzo A. Human movement analysis using stereophotogrammetry. Part 2. Instrumental errors. Gait Posture 2005;21(2):197-211.

[32] Anglin C, Wyss UP. Review of arm motion analyses. Proc Inst Mech Eng [H] 2000;214(5):541-55.

[33] Kadaba MP, Ramakrishnan HK, Wootten ME, Gainey J, Gorton G, Cochran GVB Repeatability of kinematic, kinetic and electromyographic data in normal adult gait. J Orthop Res 1989;7(6):849-60.

[34] Konczak J, Dichgans J. The development toward stereotypic arm kinematics during reaching in the first 3 years of life. Exp Brain Res 1997;117(2):34654

[35] Berthier NE, Clifton RK, McCall DD, Robin DJ. Proximodistal structure of early reaching in human infants. Exp Brain Res 1999;127(3):259-69.

[36] Berthier NE, Keen R. Development of reaching in infancy. Exp Brain Res 2006;169(4):507-18.

[37] Schneiberg S, Sveistrup H, McFadyen B, McKinley P, Levin MF. The development of coordination for reach-to-grasp movements in children. Exp Brain Res 2002;146(2):142-54

[38] Petuskey K, Bagley A, Abdala E, James MA, Rab G. Upper extremity kinematics during functional activities: three-dimensional studies in a normal pediatric population. Gait Posture 2007:25(4):573-9.

[39] Cioni G, Sales B, Poalicelli PB, Petacchi E, Scusa MF, Canapicchi R. MRI and clinical characteristics of children with hemiplegic cerebral palsy. Neuropediatrics 1999;30(5):249-55. 
[40] Staudt M, Gerloff C, Grodd W, Holthausen H, Niemann G, Krägeloh-Mann I. Reorganization in congenital hemiparesis acquired at different gestational ages. Ann Neurol 2004;56(6):854-63.

[41] Feys H, Eyssen M, Jaspers E, Klingels K, Desloovere K, Molenaers G, et al. Relation between neuroradiological findings and upper limb function in hemiplegic cerebral palsy. Eur J Paediatr Neurol 2009. doi: 10.1016/j.ejpn. 2009.01.004.

[42] van Andel CJ, Wolterbeek N, Doorenbosch CA, Veeger DH, Harlaar J. Complete 3D kinematics of upper extremity functional tasks. Gait Posture 2008;27(1): 120-7.

[43] Mosqueda T, James MA, Petuskey K, Bagley A, Abdala E, Rab G. Kinematic assessment of the upper extremity in brachial plexus birth palsy. J Pediatr Orthop 2004;24(6):695-9.

[44] Wang JS, Petuskey K, Bagley AM, James MA, Rab G. The contralateral unimpaired arm as a control for upper extremity kinematic analysis in children with brachial plexus birth palsy. J Pediatr Orthop 2007;27(6):709-11.

[45] Hingtgen B, McGuire JR, Wang M, Harris GF. An upper extremity kinematic model for evaluation of hemiparetic stroke. J Biomech 2006;3(4(9)): 681-8.

[46] Michaelsen SM, Luta A, Roby-Brami A, Levin MF. Effect of trunk restraint on the recovery of reaching movements in hemiparetic patients. Stroke $2001 ; 32(8)$ : 1875-83.

[47] Rab G, Petuskey K, Bagley A. A method for determination of upper extremity kinematics. Gait Posture 2002;15(2):113-9.

[48] Kontaxis A, Cutti AG, Johnson GR, Veeger HE. A framework for the definition of standardized protocols for measuring upper-extremity kinematics. Clin Biomech (Bristol Avon) 2009;24(3):246-53.
[49] Cappozzo A, Catani F, Della Croce U, Leardini A. Position and orientation in space of bones during movement: anatomical frame definition and determination. Clin Biomech (Bristol Avon) 1995;10(4):171-8.

[50] Leardini A, Chiari L, Della Croce U, Cappozzo A. Human movement analysis using stereophotogrammetry. Part 3. Soft tissue artifact assessment and compensation. Gait Posture 2005;21(2):212-25.

[51] Karduna AR, McClure PW, Michener LA. Scapular kinematics: effects of altering the Euler angle sequence of rotations. J Biomech 2000;33(9):1063-8.

[52] Senk M, Chèze L. Rotation sequence as an important factor in shoulder kinematics. Clin Biomech (Bristol Avon) 2006;21(Suppl. 1):S3-8.

[53] Morrow DA, Lin HT, Kaufman KR, An KN. Clinically significant shoulder joint kinematics description. Gait Posture 2006;24(Suppl. 2):S45-7.

[54] Rab G. Shoulder motion description: the ISB and Globe methods are identical. Gait Posture 2008;27(4):702-5.

[55] Mottram SL. Dynamic stability of the scapula. Man Ther 1997;2(3):123-31.

[56] Garofalo P, Cutti AG, Filippi MV, Cavazza S, Ferrari A, Cappello A, et al. Interoperator reliability and prediction bands of a novel protocol to measure the coordinated movements of shoulder-girdle and humerus in clinical settings. Med Biol Eng Comput 2009;47(5):475-86.

[57] Meskers CGM, van der Helm FCT, Rozendaal LA, Rozing PM. In vivo estimation of the glenohumeral joint rotation center from scapular bony landmarks by linear regression. J Biomech 1998;31(1):93-6.

[58] Stokdijk M, Nagels J, Rozing PM. The glenohumeral joint rotation centre in vivo. J Biomech 2000;33(12):1629-36.

[59] Schmidt R, Disselhorst-Klug C, Silny J, Rau G. A marker-based measurement procedure for unconstrained wrist and elbow motions. J Biomech 1999;32(6): 615-21. 